\title{
COVID-19 patients and the radiology department - advice from the European Society of Radiology (ESR) and the European Society of Thoracic Imaging (ESTI)
}

\author{
Marie-Pierre Revel ${ }^{1} \cdot$ Anagha P. Parkar ${ }^{2,3} \cdot$ Helmut Prosch $^{4} \cdot$ Mario Silva $^{5} \cdot$ Nicola Sverzellati $^{5} \cdot$ Fergus Gleeson $^{6}$. \\ Adrian Brady ${ }^{7}$ on behalf of the European Society of Radiology (ESR) and the European Society of Thoracic Imaging (ESTI)
}

Received: 24 March 2020 / Revised: 1 April 2020 / Accepted: 2 April 2020 / Published online: 20 April 2020

(C) European Society of Radiology 2020

\begin{abstract} (e.g. oncology, other infectious diseases etc.), will be discussed.

\section{Key points}

- Bilateral ground glass opacities are typical CT manifestations of COVID-19.

- Crazy paving and organising pneumonia pattern are seen at a later stage.

- Extensive consolidation is associated with a poor prognosis.
\end{abstract}

This document from the European Society of Radiology (ESR) and the European Society of Thoracic Imaging (ESTI) aims to present the main imaging features, and the role of CT scan in the early diagnosis of COVID-19, describing, in particular, the typical findings which make it possible to identify the disease and distinguish it from bacterial causes of infection, and to define which category of patients may benefit from CT imaging. The precautions that must be taken when performing scans to protect radiologists and technologists from infection will be described. The organisational measures that can be taken within radiology departments in order to cope with the influx of patients, while continuing to manage other emergency and time-sensitive activity

Keywords Coronavirus infections · Pneumonia · Tomography, X-ray computed

Abbreviations

COVID-19 Corona virus disease 19

Marie-Pierre Revel

marie-pierre.revel@aphp.fr

Adrian Brady

adrianbrady@me.com

1 Department of Radiology, Cochin Hospital, Université de Paris, 75006 Paris, France

2 Department of Radiology, Haraldsplass Deaconess Hospital, Bergen, Norway

3 Department of Clinical Medicine, Faculty of Medicine and Dentistry, University of Bergen, Bergen, Norway

4 Department of Biomedical Imaging and Image-guided Therapy, Medical University of Vienna, Vienna, Austria

5 Radiology, Department of Medicine and Surgery, University of Parma, Parma, Italy

6 Department of Oncology, University of Oxford, Oxford, UK

7 Department Radiology, Mercy University Hospital, Cork, Ireland
$\mathrm{CT}$
ECDC
Computed tomography
PPE
European Centre for Disease Prevention and Control

RT-PCR Reverse-transcription polymerase chain reaction

The outbreak of SARS-CoV 2, a new coronavirus, has rapidly spread worldwide since the end of 2019, affecting most European countries in March 2020, with Italy, Spain and France being the most severely affected to date. This viral infection is responsible for a respiratory disease, corona virus disease 19 (COVID-19), that can be very severe.

While confirmation of the diagnosis relies on the identification of viral RNA by the reverse-transcription polymerase chain reaction (RT-PCR), variability in the processing of swabs may delay results. In such a setting, another solution with sufficient accuracy is needed to guide the quick management of patients presenting to hospitals during this pandemic. Radiologists can help in this task by identifying and characterising pulmonary involvement of COVID-19 [1]. 

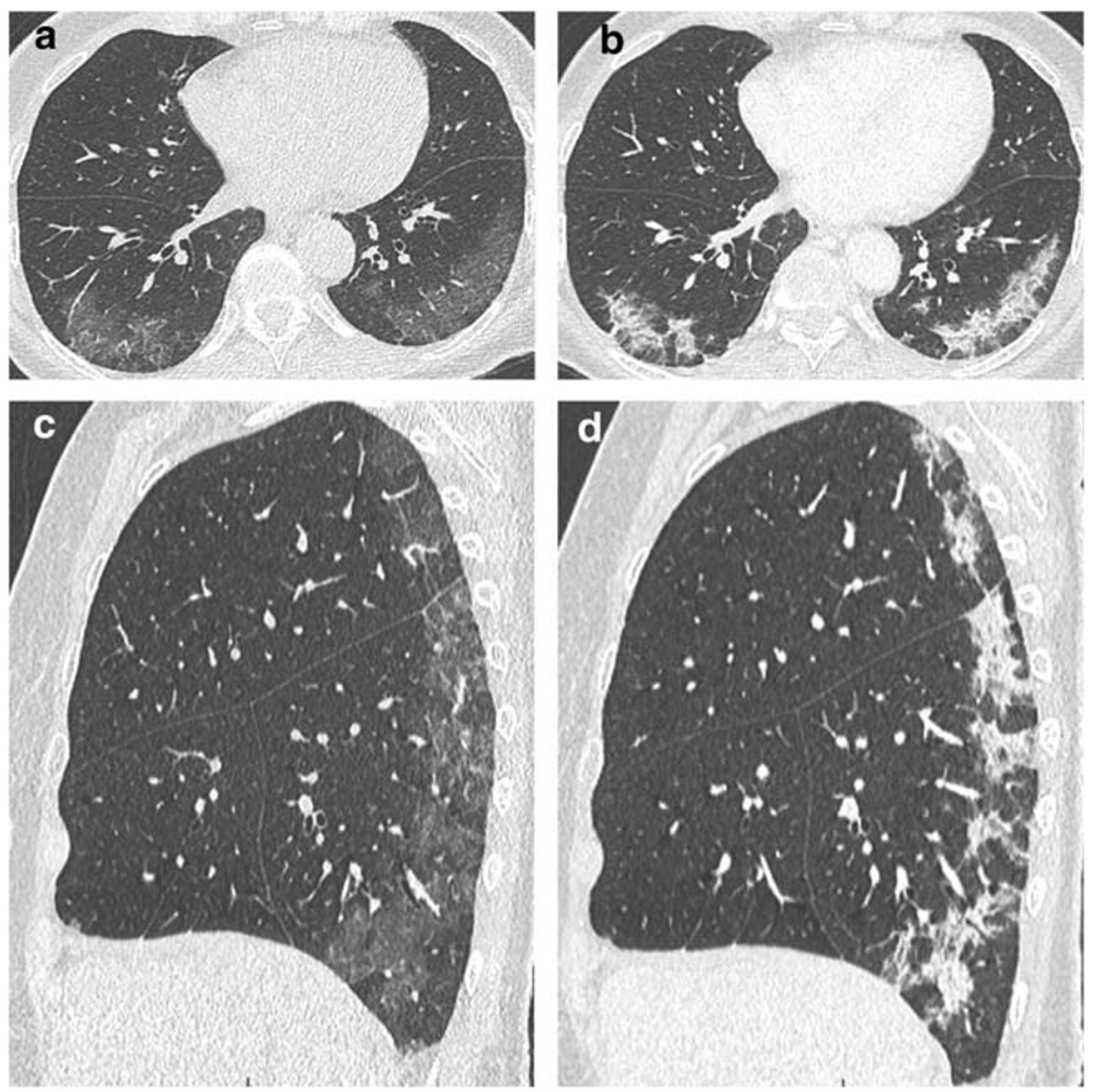

Fig. 1 Axial and sagittal thin-sections showing bilateral pure ground glass in the sub pleural portion of both lower lobes, at presentation, in a 71-year-old woman with cough and fever for 5 days $(\mathbf{a}, \mathbf{c})$. Evolution 6 days later with linear consolidation typical for organizing pneumonia pattern (b, d)

Despite the non-specific findings of diffuse alveolar damage and the variable extent of organizing pneumonia that are generally seen in viral infections, the specific nature of this pandemic makes imaging a good and accurate tool to stratify patients selected from first-line clinical triage. Additionally, a large series based on 1,014 Chinese patients reported a higher sensitivity for chest computed tomography (CT) compared to RT-PCR, with a mean interval time between the initial negative to positive RT-PCR results of $5.1 \pm 1.5$ days [2].

\section{Which imaging techniques can be used when COVID-19 pneumonia is suspected, and in COVID-proven patients?}

Chest radiography is not sensitive for the detection of ground glass opacities, which are the main imaging features of COVID19 pneumonia. It should not be used as the first-line technique and should be restricted to the follow-up of patients admitted to intensive care units, who are too fragile to be sent for CT.

Chest ultrasound does not allow differentiation between bacterial and viral pneumoniae, nor between pulmonary oedema and infection. Ultrasound is used at the bedside to diagnose complications such as a pneumothorax under mechanical ventilation or pleural effusions and can help in adjusting mechanical ventilation or monitoring pulmonary fluid load.

Ultrasound use for other organs should be limited to indications where it is likely to be definitive in its findings. If US is unlikely to be diagnostic, CT should be preferred (even in circumstances where US might be used first in ordinary circumstances), to minimise staff exposure. CT use must be balanced by the knowledge that those staff engaged in patient transportation to the CT unit may be exposed, and must be protected. Where US is appropriate and needed (e.g. pregnant women, children, ICU patients), it should ideally be performed at the bedside, to avoid patient transport and consequent staff exposure.

\section{What are the main CT features of COVID-19?}

In the early phase of the disease, CT typically shows bilateral ground glass opacities, with a predominantly peripheral, subpleural location [3-5]. These opacities can be quite extensive in the sub-pleural areas (Figs. 1a, c, 2a and 3) or may be more focal, with a rounded shape (Fig $2 b$ ). Intralobular reticulations can be seen superimposed on the ground glass opacities, resulting in a crazy-paving pattern (Fig. 2e) which is usually associated with a more severe stage of the disease. Patients evaluated several days after the beginning of 
Fig. 2 Typical findings of COVID-19 pneumonia in CT Ground glass opacities in large area $(\mathbf{a})$ or nodular $(\mathbf{b})$. Consolidation focal (c) or linear (d). Crazy paving pattern (e)
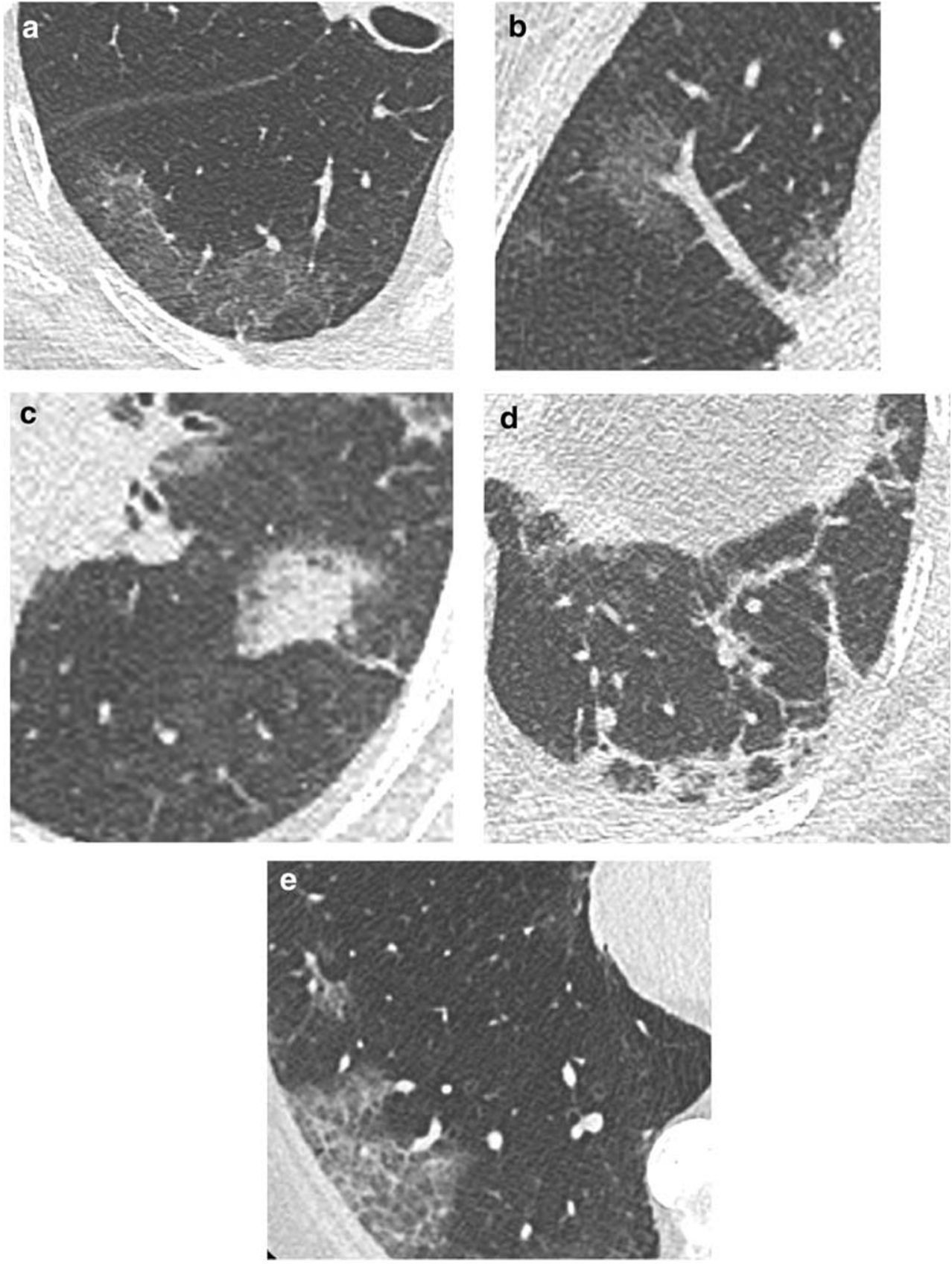

symptoms can present with linear consolidation (Figs. 1b, d and 2d) or areas with reverse halo appearances, both suggesting organizing pneumonia, which is a common reaction to lung injury [6]. Extensive consolidation is associated with a poorer prognosis in older patients (Fig 4c).

Significantly, mucoid impactions, centrilobular nodules, lobar consolidation, lymphadenopathy or significant pleural effusions (Fig. 5) are infrequently seen in COVID-19. Consequently, these findings suggest alternative diagnoses or complications such as super-added bacterial infection or heart failure.

It is important to be aware that chest $\mathrm{CT}$ has been reported to demonstrate no abnormalities in the first 3 days of symptoms (day 0 - day 2) in $56 \%$ of patients [7]. Conversely, patients without symptoms may show abnormal findings on CT (albeit with fewer lung segments involved than in symptomatic patients) [8].

\section{Who should benefit from CT and what information can be obtained?}

CT should not be performed as a screening test in patients with mild or no symptoms. It is indicated after the clinical evaluation of patients with respiratory symptoms such as dyspnoea and desaturation, the degree of severity justifying investigation being left to clinical judgement and depending on local resources. CT may also be helpful in patients with milder 

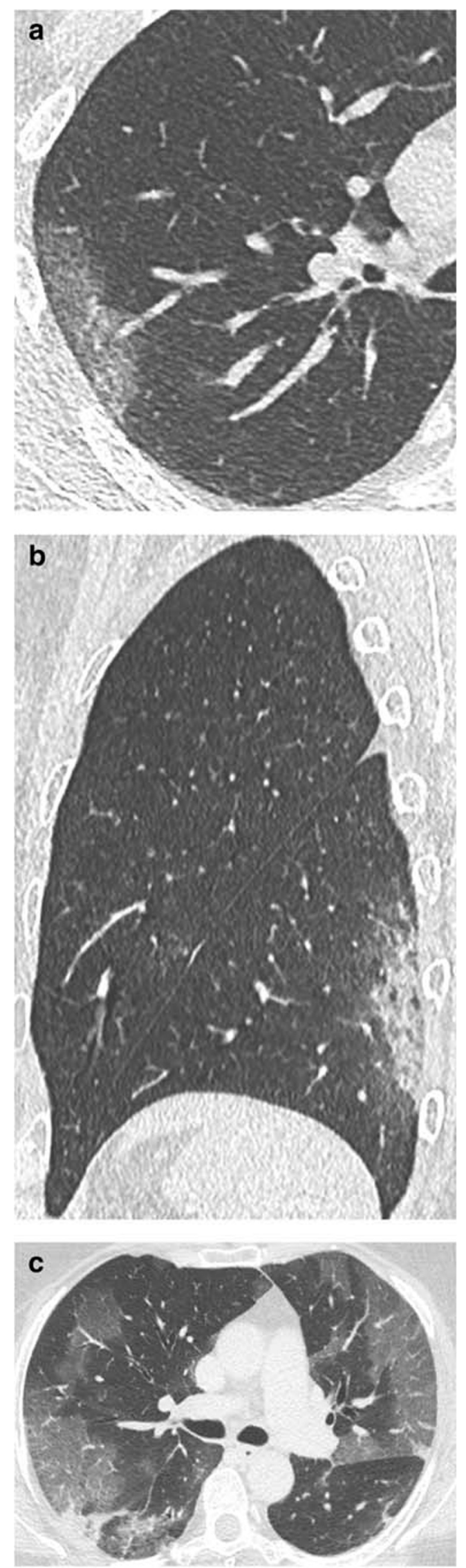

Fig. 3 Subpleural predominance (a-c)
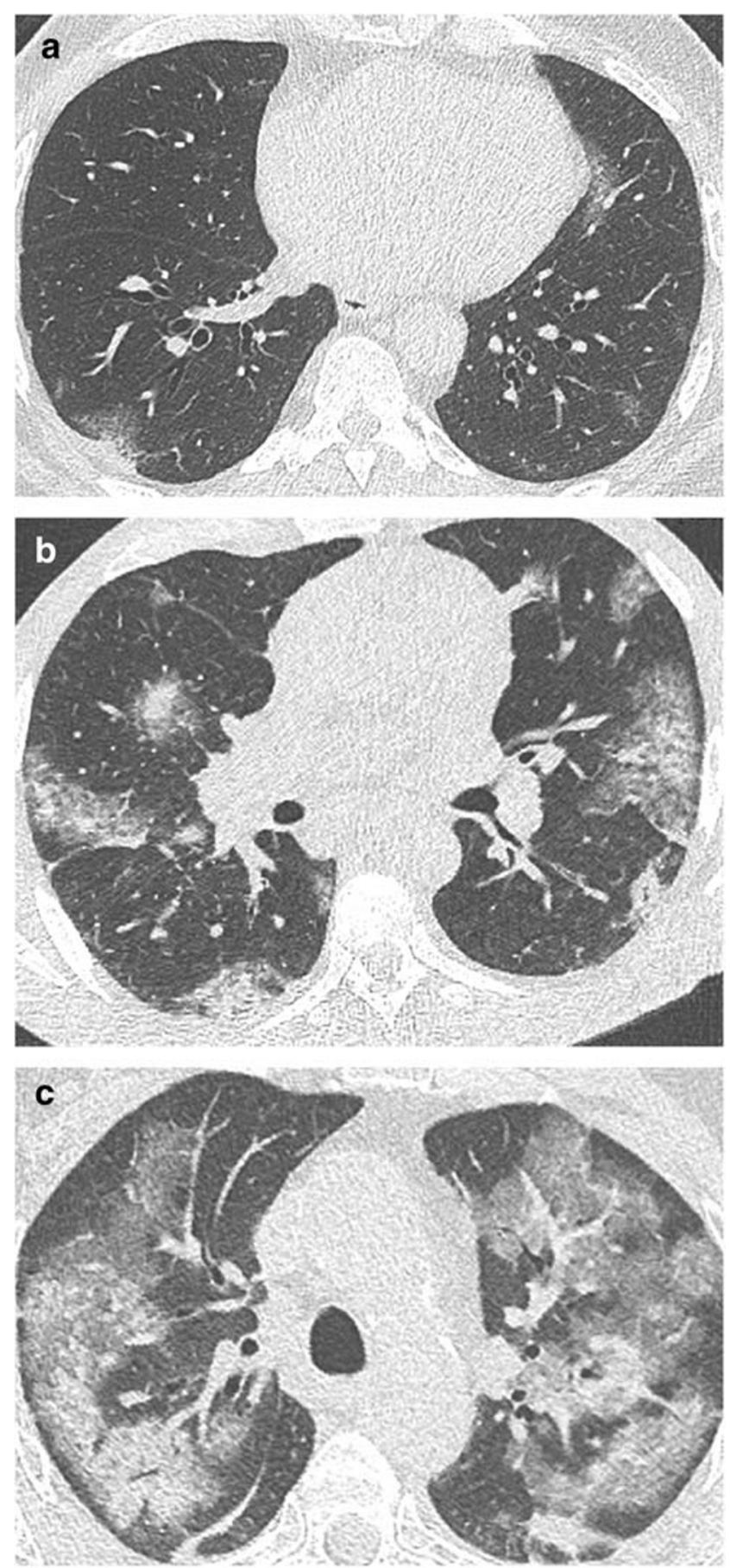

Fig. 4 Disease extent mild (a), moderate (b), severe (c)

symptoms who have co-morbidities, such as diabetes, obesity, chronic respiratory disease, etc. It is important to note that although the CT findings of COVID-19 are characteristic, the final diagnosis requires a positive RT-PCR test.

In the case of an initially negative RT-PCR and CT changes highly suggestive of COVID-19, the RT-PCR should be repeated to determine if it had been a false-negative result. The sensitivity of the RT-PCR depends on which test has been used, the quality of the throat swab and the intensity of viral load. 
Fig. 5 CT features suggesting other diagnoses. Mucoid impactions (a), tree-in-bud (b), centrilobular nodules (c), septal lines (d), segmental consolidation (e)
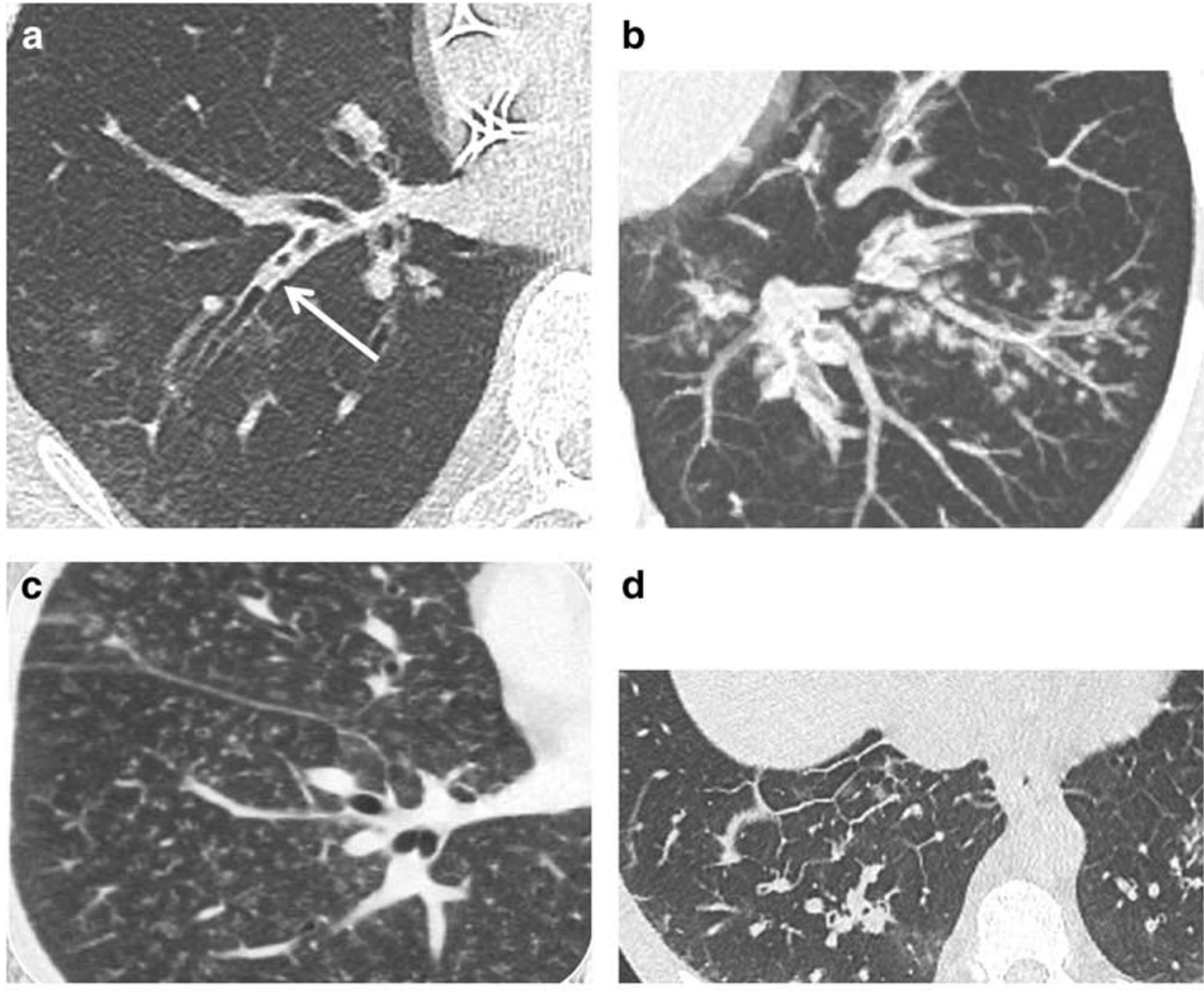

\section{d}

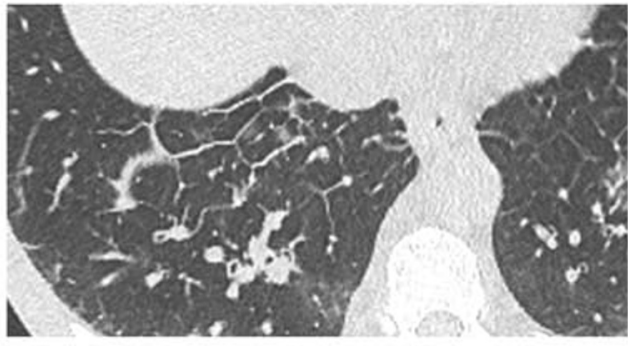

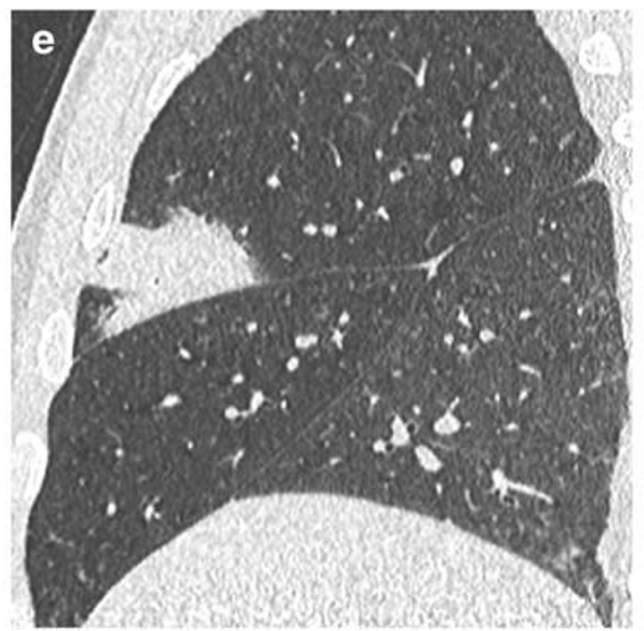

CT allows evaluation of disease extent at baseline, which may help to predict a poor outcome and the need for ventilation. If supplementary oxygen is needed in patients with limited disease extension, other diagnoses, especially pulmonary embolism, should be suspected and an additional contrastenhanced CT acquisition may be indicated. Lastly, CT allows for the identification of signs of pulmonary oedema, raising the suspicion of COVID-19 related myocarditis, in which case troponin measurement and echocardiography may be required.

Repeat CTs are indicated in cases with suspected complications (e.g. superinfection, pulmonary embolism). As the transport of patients from the ward to the CT unit is associated with the risk of contamination of other patients and personnel, the indications for repeat scans should be considered carefully. In patients that are recovering, a repeat $\mathrm{CT}$ is not necessary.

\section{What protective measures should be taken for the staff?}

The recommendations of the European Centre for Disease Prevention and Control (ECDC) should be followed [9]. Close collaboration with hospital departments of Infection 
Control is appropriate; these departments will usually provide hospital-wide guidance on protective measures.

For any health care professional with patient contact it is noted that nail polish, and rings are prohibited, and it is recognised that beards decrease FFP2 mask efficacy.

The minimal personal protective equipment (PPE) includes face masks, goggles (or a face shield), gloves and a longsleeved gown. The procedure for dressing and undressing has a specific order, and everyone must familiarise themselves with it. The order is: 1) hand wash, 2) don the long-sleeved gown, 3) fix the gown at the back, 4) put on and fix the face mask,5) put on the goggles, 6) put on gloves. The removal of the PPE is done in reverse order. After removing the gloves, a new pair of gloves is recommended to avoid contamination during disrobing. For further instruction see [9].

When performing a CT, the number of staff in direct contact with the patient should be reduced. If possible, only one technologist should be in direct contact with the patient in the CT room and another technologist should operate the CT scanner. Both technologists should wear masks. The patient should be brought directly into the CT room. The technologist managing patient positioning on the CT table should wear disposable gloves, long-sleeved gown and goggles.

The ECDC recommends that a particular respirator mask (such as EU standard FFP2) should be used when performing aerosol generating procedures. The WHO list of aerosol generating procedures includes tracheal intubation, non-invasive ventilation, manual ventilation prior to intubation and bronchoscopy [10].

\section{How should imaging departments organise to face the epidemic?}

\section{Procedures}

Non-urgent procedures such as systematic evaluation of chronic diseases and screening procedures (e.g. mammography) should be postponed. Management of general imaging emergencies should be maintained for in- and out-patients, including the evaluation of acute worsening of chronic disease. Imaging activity related to the management of oncologic patients should be maintained, especially the initial diagnosis and staging, including percutaneous biopsies, response evaluation during chemotherapy and other treatments.

\section{Staff}

Direct staff exposure to infected patients should be controlled, such that proper patient care is provided, but nobody is exposed unnecessarily. Thus, staff present in the radiology department should be kept to a minimum, with those who can do so working from home, and others being deployed to areas in the hospital which may need additional staff. Reinforcement of the technologist team for bedside chest radiographs is needed for the management of intensive care patients. More radiologists and technologists are needed for night and weekend shifts and fewer for usual hours.

Trainee radiologists and radiologists not usually involved in chest imaging should be educated to recognise the typical radiologic findings that may be expected in COVID patients.

An inventory of staff skills and training should be maintained, to ensure that staff can be re-deployed as necessary to areas within and outside the radiology dept. where shortages develop, or demand increases.

\section{Spatial organisation}

Whenever possible, the imaging department should dedicate a specific area (including waiting space) and specific equipment (CT scanner, plain radiography room etc.) to the evaluation of patients with suspected or confirmed COVID-19, to minimise the risk of contamination of other patients and concentrate the protective material availability.

In some hospitals, the use of a mobile CT unit close to the location where clinical triage is performed at the entrance to the hospital might be considered, depending on the availability of access to standard CT equipment.

\section{Communication}

Communication with radiology dept. and other hospital staff, such as through a daily newsletter or briefing, is essential to provide the latest information about the workflow depending on the current situation at the hospital. Safety measures should be regularly reinforced and the procedures put in place for staff members suspected of having COVID (and for how they should be replaced to maintain workflow) should be clearly explained. Communication with other departments is also essential to revise and update internal guidelines, as workflows may need to be adjusted to a rapidly changing situation.

\section{Structured report proposal}

\section{STRUCTURED REPORT PROPOSAL (for lung parenchymal findings in possible COVID)}

Unenhanced thin-section chest CT performed for COVID-19 suspicion/initial assessment/follow-up

DLP: (mGy.cm)

\section{Findings}

- Presence of (bilateral, diffuse, confluent, patchy) ground glass opacities with /a rounded morphology/a crazy 
paving pattern/a peripheral distribution without subpleural sparing

- Presence of ground-glass opacities admixed with perilobular consolidations /linear consolidation

- Presence*/Absence of tree-in-bud pattern/centrilobular nodules/endobronchial secretion/lobar or segmental consolidation

- Presence*/Absence of adenopathy/significant pleural effusion

Conclusion CT findings (highly/indeterminate/non) suggestive of COVID 19, with limited, moderate, severe disease extent $^{\circ}$

*: if present, suggestive of other infectious disease of superinfection

${ }^{\circ}$ : based on visual assessment. A scale $(<10 \%, 10-25 \%, 25-$ $50 \%, 50-75 \%,>75 \%$ ) may be used.

Other structures such as heart and vasculature, pleura, mediastinum and bony and soft tissues should be evaluated as usual.

When CT findings are typical, recognising and identifying the COVID-19 pattern will enable swifter recognition of COVID patients and aid clinicians to triage these patients. Radiologists must be aware of these findings, and must make the effort to indicate their level of confidence of the presence or absence of typical COVID findings in their reports. Although it may sometimes be necessary to use the "indeterminate" category, this should be avoided as far as possible, as it limits the clinical usefulness of the report.

\section{Conclusion}

The COVID 19 pandemic poses unprecedented challenges for provision and maintenance of radiology services, and for protection of staff and non-infected members of the public. The experiences of those countries which have been earliest affected can help to guide those for whom the peak of infection and disruption comes later. In this paper, we seek to offer simple guidelines for radiologists and radiology departments to assist in meeting the demands of this crisis.

Acknowledgements This paper was prepared by representatives of the European Society of Radiology (ESR) and the European Society of Thoracic Imaging (ESTI). It was endorsed by the ESR Executive Council and the ESTI Executive Committee in March 2020.

Funding information The authors state that this work has not received any funding.

\section{Compliance with ethical standards}

Guarantor The scientific guarantor of this publication is Marie-Pierre Revel.

Conflict of interest The authors of this manuscript declare no relationships with any companies, whose products or services may be related to the subject matter of the article.

Statistics and biometry No complex statistical methods were necessary for this paper.

Informed consent Written informed consent was not required for this study because this is a recommendation paper.

\section{References}

1. $\operatorname{Kim~H}$ (2020) Outbreak of novel coronavirus (COVID-19): what is the role of radiologists? Eur Radiol. https://doi.org/10.1007/ s00330-020-06748-2

2. Ai T, Yang Z, Hou H et al (2020) Correlation of chest CT and RTPCR testing in coronavirus disease 2019 (COVID-19) in China: a report of 1014 cases. Radiology. https://doi.org/10.1148/radiol. 2020200642

3. Ye Z, Zhang Y, Wang Y, Huang Z, Song B (2020) Chest CT manifestations of new coronavirus disease 2019 (COVID-19): a pictorial review. Eur Radiol. https://doi.org/10.1007/s00330-020-06801-0

4. Wu J, Wu X, Zeng Wet al (2020) Chest CT findings in patients with Corona virus disease 2019 and its relationship with clinical features. Invest Radiol. https://doi.org/10.1097/RLI.0000000000000670

5. Zhao W, Zhong Z, Xie X, Yu Q, Liu J (2020) Relation between chest $\mathrm{CT}$ findings and clinical conditions of coronavirus disease (COVID-19) pneumonia: a multicenter study. AJR Am J Roentgenol 3:1-6

6. Pan $Y$, Guan $H$, Zhou S et al (2020) Initial CT findings and temporal changes in patients with the novel coronavirus pneumonia (2019nCoV): a study of 63 patients in Wuhan, China. Eur Radiol. https:// doi.org/10.1007/s00330-020-06731-x

7. Bernheim A, Mei X, Huang M et al (2020) Chest CT findings in coronavirus Disease-19 (COVID-19): relationship to duration of infection. Radiology. https://doi.org/10.1148/radiol.2020200463

8. Shi H, Han X, Jiang N et al (2020) Radiological findings from 81 patients with COVID-19 pneumonia in Wuhan, China: a descriptive study. Lancet Infect Dis 20(4):425-434. https://doi.org/10. 1016/S1473-3099(20)30086-4

9. European Centre for Disease Prevention and Control (2020) Guidance for wearing and removing personal protective equipment in healthcare settings for the care of patients with suspected or confirmed COVID-19. https://www.ecdc.europa.eu/sites/default/ files/documents/COVID-19-guidance-wearing-and-removingpersonal-protective-equipment-healthcare-settings-updated.pdf. Accessed 23 March 2020

10. World Health Organization (2020) Advice on the use of masks in the community, during home care and in healthcare settings in the context of the novel coronavirus (2019-nCoV) outbreak: interim guidance, 29 January 2020. World Health Organization. https:// apps.who.int/iris/handle/10665/330987. Accessed 23 March 2020

Publisher's note Springer Nature remains neutral with regard to jurisdictional claims in published maps and institutional affiliations. 